\title{
Physical Energetics of Photons and New Technical and Social Possibilities of Mankind
}

\author{
OSTAPENKO V. A. \\ Acad. UkrAS, Ukraine, Kiev;
}

The concept of the new managed Eco-Physical hydrogen Energetics of Photons (EFhEF - energetics) is introduced. The new set of available data became the basis for classification of energy: "the energetics of molecules (chemical), atoms (physical), and nuclei (quantum). The $E F h E F$ - energetics can be used together with chemical and thermonuclear energetics on the Earth and jointly with thermonuclear energetics outside the Earth. The physical energetics has been introduced as the result of the theoretical and experimental researches of active processes in solar plasma. The problem of Sun's flare activity has been solved. In the first time, in the Nature, the current sheets have been discovered, which in able of to transform the magnetic energy into energy of plasma.For the first time, the $E F h E F$ energetics provided any installation by its own source of energy. The energy is released as photons of continuous radiation of negative hydrogen ion (without oxygen and fossil fuels). It is identical to the radiation of the photosphere of the Sun. Namely this energy is the basis of all life (as flora so fauna) on the Earth. This allows to actually inheriting the rich experience of the wildlife for operation with organic matter. The physical energetics can solve the problems of ecology and of the replacement of fossil fuels that means and the solution of primary problem of the energetics.

Wildlife takes full advantage of all (known and unknown for us) physical and chemical properties of atoms and molecules to create of the organics. The photosynthesis only is the way to capture $\mathrm{CO}_{2}$ and of Sun's energy to ensure of organic structures. The wildlife is managed by the software, recorded in the DNA, and works in terrestrial atmospheric conditions $\left(4-40^{\circ} \mathrm{C}\right)$ using the energy of solar photons. We may to inherit the technologies of wildlife (yet photosynthesis) which use energy in the form of separate photons of solar radiation. And it is able to meet the challenges of life outside the Earth. These are lighting and heat, air and water regeneration, and return of all $100 \%$ organic waste at the new cycle of use, and even with the production of food from these waste. The new energetics allows "to carry out" even our Sun at any distance in the space. The creation technologies of living organisms are controlled in details by the "numeric" programs, written in the DNA molecules. The nucleotides are the basis unit of information and the primary building blocks of all subsequent organic structures. Also the catalysts (enzymes, pigments) or, in other words, atoms properties (metals and their oxides) are wide used.

The energetics of photons has been revised on the Earth by the live Nature during some billion years. Our task is to inherit this rich experience. This new physical and environmental energetics, which is by full analogue of solar radiation, which changes of the life quality, and which may be obtained by people, is not yet used on the Earth. It provides the need to discuss together researchers, professors, and high education students of different aspects of new possibilities in life quality, and to introduce these researches in all fields of education, social sciences and humanities.

Key words: the Sun's flares, solar radiation, new physical energetics, hydrogen plasma, environment, organics decomposition, replacement of natural fuels, photosynthesis.

\section{THE CHEMICAL (MOLECULES), PHYSICAL (ATOMS) AND QUANTUM (NUCLEI) ENERGETICS}

Today humanity meets its energy needs, mainly by burning oil, gas and coal. However, affordable and cheap their supplies are limited. With the growth of population and the alignment of the quality of life in its various regions is increasing energy consumption. According to projections, it is expected that the population of the Earth to the middle of the 21st century will increase to 9-10 billion people. Estimates show that by this time, world energy consumption will increase by several times. This increase in energy consumption on the planet need to provide sources of energy commodities, and the solution to this problem, start now, because the widespread introduction of new technologies in energy production takes more than a dozen years.

Biofuels (of organic waste) in the form of firewood, humanity uses for centuries, and hydrogen energy today seems to be the most promising! But, natural deposits of hydrogen on the Earth are missing. There are two ways to get hydrogen on industrial scales. This is either the decomposition of water into hydrogen and oxygen by electrolysis. And it requires energy, which surpasses yet the burning hydrogen energy when converting it back into the water. Either get hydrogen from fossil gas using the catalysts and burning fossil fuels! 
Electricity is of accessible and familiar means of delivery and use of energy. It is thanks to electricity, mankind has made such a big spurt in its progress for $~ 100$ years. But thermal power stations use the limited fossil fuels. The Tokomak and ITER are reactors with magnetic plasma confinement for to achieving the necessary conditions for controlled thermonuclear fusion and obtain of energy. In the vacuum chamber, nuclei of deuterium and tritium colliding together created the helium nuclei (alpha particles) and also high-energy neutrons. Plasma in the Tokomak is confined by external toroidal and poloidal magnetic fields, which created by electricity. After of our discovery of the current sheets as solar flares, the Project of the Delaware University "Plasma physics' "giant leap" is beginning (Shay M. et al., 2016). The aim of the project is to understand magnetic reconnection, a process that produces powerful phenomena including solar flares.

Magnetic plasma confinement found in the Sun (Ostapenko, 2011, ru; 2012, en). Solar flare has appeared to be the current sheet $(C S)$ directly. Namely the current sheet, which is formed when magnetic fields are clashed, have confined the plasma and have defined all of its physical and dynamic properties and manifestations. The observed current sheets are investigated experimentally and theoretically by the author. Solar flares and their evolution is the process of collision of magnetic fields, like of meteorites in the Earth's atmosphere. The conditions of formation and destruction of the current sheets are found. Data from these studies are directly related to the problems of magnetic plasma confinement in thermonuclear fusion in installations of Tokomaks-type, as well as in laboratory operation with current sheets.

Physical (of atoms) energetics provides cost-effective production of hydrogen fuel, the fuel of ecological and efficient. We have developed the new technology of physical decomposition (not burning) of organic nature $\left(\mathrm{H}_{n} \mathrm{C}_{m} \mathrm{~N}_{j} \mathrm{O}_{k}\right.$ molecules) into hydrogen $\left(\mathrm{H}_{2}\right)$, carbon $(\mathrm{C})$ and atmospheric atoms $\left(\mathrm{O}_{2}+N\right)$. The organics decomposition occurs in closed reactors and is also accompanied by the emergence of synthesis gas. His appearance is due to the presence of the atmospheric nitrogen and oxygen in the reaction zone. The processes of destruction and the emergence of synthetic gas molecules $\left(\mathrm{H}_{2} \mathrm{O}, \mathrm{CO}_{2}, \mathrm{CO}\right.$, and $\left.\mathrm{CH}_{4}\right)$ occur continuously. But our synthesis gas go through yet the process of synthesis up to a maximum amount of gas or/and gasoline and of carbon/graphenes.

Negative ion of hydrogen is of great importance in the field of atomic physics, astrophysics, and wildlife. Our calculations revealed managing of release intensity this energy in any volume of the plasma. The technology allows creating of "solar" photons for lighting, heating or inside of reactors. This allows providing the "soft" technologies of decomposition or synthesis of organic matter. And photosynthesis of plants in terrestrial atmosphere goes with a maximum effect at $25^{\circ} \mathrm{C}$.

\section{MANAGED PHYSICAL ENERGY OF HYDROGEN PLASMA AND SOLAR FLARES}

Continuous radiation of negative hydrogen ion turned out to be manageable (fig.3) that can be used for terrestrial applications. This is the new physical and environmental form of energy, which on the Earth is not yet used as such. The EFhEF (Eco Physical hydrogen Energetics of Photons) originated on the basis of the analysis of hydrogen plasmas in the annex to the active processes (solar flares) on the Sun. The EFhEF-energetics believes operating with the single photons as energy sources for detailed processes of synthesis or decomposition of organic matter. A task made easier by the fact that this energetics has already been mastered in the Nature for the synthesis of the organic matter (photosynthesis). These technologies are working on the Earth billions of years already, and it giving us hopes of success. And only managed physical EFhEF - energetics [1] that inherits the photosynthesis technologies indicates the development ways of such technologies of decomposition/synthesis of the organics. The problem of creating a "solar" field of radiation in the installation reactor is similar on the Earth already with problems of creating "artificial Sun" ([6], section 1-7). In our case, it is need to create hydrogen-gas and it is succeed to excite into install the negative ion of hydrogen with its "solar" radiation. Radiation of solar photosphere is provided by excitation of negative hydrogen ions. Spectral distribution of solar radiation has a maximum in green $(550 \mathrm{~nm})$ range. It is to this region adapted the eye of the animal world. A molecule of chlorophyll absorbs red (and IR) and blue (and the near UV) photons spectrum (fig.1b) and scatters radiation green area of the spectrum. The degree of negative hydrogen ion excitation is determined only by the plasma density of hydrogen atoms in the volume of plasma and occurs without the participation of oxygen. Theoretical dependency on fig. 1c gives us the ability to manage the process of release of energy. Problems of high temperatures $\left(6000^{\circ} \mathrm{K}\right.$ in photosphere) cut by not-thermal excitation mechanisms of hydrogen plasma. A task made easier by the need to create only UV part (2500-3500 Á) of Planck curve (fig.4). Magnetic confinement problem in such plasma $\left(\sim 0^{\circ} \mathrm{C}\right)$ simply does not arise.

We selected the adaptation task of the EFhEF- energetics to its capability to the physical decomposition (not burning) of any organic matters. The first installations of the decomposition of organic matter have already shown its effectiveness. When the gas-fuel is produced by our technologies, would mean and the decision of the principal problem of the energetics. This is the problem of replacing fossil energy sources of unrestricted organic wildlife. Only the EFhEF - energetics is capable to decompose selectively the polluting spills of 
petroleum products, as well as to separate radioactive impurities from organic matter obtaining of pure synthesis gas $\left(\mathrm{H}_{2}, \mathrm{H}_{2} \mathrm{O}, \mathrm{CO}, \mathrm{CO}_{2}, \mathrm{CH}_{4}\right.$, and $\left.\mathrm{C}_{n} \mathrm{H}_{2 n}\right)$ or gas-fuel. Other enabling technologies of the $E F h E F$ - energetics: 1 . Disposal of pesticides and toxic chemicals. 2. Utilization of polyethylene and plastics. 3. The air and water regeneration systems. 4. Elimination of atmospheric ejections of heat boilers with th production of gas-fuel.

The process of decomposition of organic molecules $\left(\mathrm{H}_{n} \mathrm{C}_{m} \mathrm{~N}_{j} \mathrm{O}_{k}+\mathrm{H}_{2} \mathrm{O}\right)$ to combustible (synthesis-) gas fuel $\left(\mathrm{H}_{2}+\mathrm{C}_{n} \mathrm{H}_{2 n+2}+\mathrm{H}_{2} \mathrm{O}+\mathrm{CO}+\mathrm{CO}_{2}\right)$ occurs on the scheme $\left(\mathrm{H}_{n} \mathrm{C}_{m} \mathrm{~N}_{j} \mathrm{O}_{k}+\mathrm{H}_{2} \mathrm{O}+\right.$ photons + catalysts $)$ like of photosynthesis schemes $\left(\mathrm{CO}_{2}+\mathrm{H}_{2} \mathrm{O}+\right.$ photons + chlorophyll). It is no burning; it is the decomposition of the organics), and always with the production of the gas fuel.

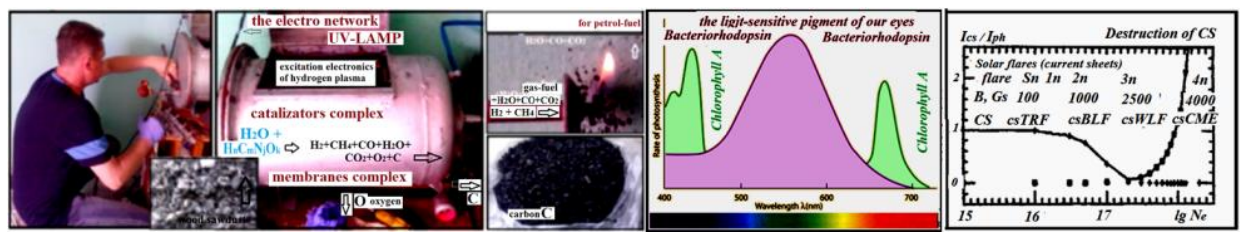

Fig.1. Managed physical energy of hydrogen plasma in laboratory

$a$. The laboratory installation of the decomposition of organic matter and the production of hydrogen-hydrocarbons gasfuel. Our installation is equipped with next systems: (a) electronics for generation of "solar" photons; (b) generation of protons, thermal and accelerated electrons; (c) systems of water photolysis; (d) catalysts systems for decomposition of organics and synthesis of new organic compounds; (e) membrane systems for selection of some gas components.

On the figure (left) Igor V. Ostapenko is

Director of the laboratory of EFhEF- energy. $\boldsymbol{b}$. the corpuscular-wave duality in the absorption curves of the chlorophyll molecule:

-UV photons excited of carbon atoms electrons accumulating energy of these photons in the $C-H$ links of organic molecules.

-IR photons (waves already) heated only organic matter (increasing the molecule's controllability) [7]. $c$. The calculated intensity curve of energy release in the form of hydrogen plasma. It is defined only by the plasma density. The curve has reflected of all solar flares emission. So, the main problem of solar flares is proved to be resolved by Ostapenko [1].

\section{PHYSICAL ENERGETICS OF PHOTONS SERVES OF THE BASE OF LIVE' NATURE}

Bioenergetics (of atoms and biomolecules) also is manageable, but programmatically. Nucleic acids are the building blocks of the information contained in DNA, in order to ensure the structure and processes of live organisms. As primary structure, the polynucleotide chain is secreted in the structural organization of the DNA molecule. The secondary structure, two complementary chains connected by hydrogen links are marked two complementary to each other chains. The formed the tertiary structure-three-dimensional spiral with defined spatial characteristics. The genetic information written in the DNA is decoded at incredible speeds of the messenger (RNA molecules). The RNA recognizes the "letters" of the DNA and is constructed of them three element "words". Namely these groups of three letters make up the biological and chemical basis of all life forms on the Earth. And all twenty amino acids, which form a set of all protein compounds of our planet, are "written" namely from these three letters "words" (codons). Codons are translated of ribosome and tRNA in the sequences of amino acids.
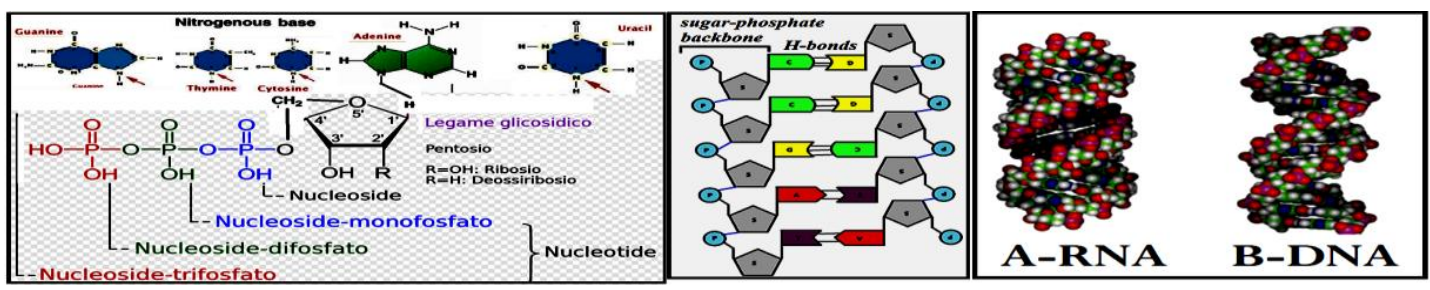

Fig.2. Nucleotides are the most important part of organic compounds of non-protein nature.

hey are components of enzymes and other biologically active substances; they serve as vectors of energy in the cells. The nucleotide into the DNA or the RNA is created in the form of one residue of phosphoric acid.

c) The system of the DNA amino acids coding on the base of nucleotides (see, also,

fig.3).http://www.biosyn.com/tew/purines-pyrimidines-and-nucleotides.aspx

Molecule of each (mono) nucleotide consists of three parts, which are chemically different. 1. This is a fivecarbon sugar (pentose). 2. The Nucleoside creates purine base or pyramidine base connected to the carbon atom of the sugar. 3. The nucleotide into the DNA or the RNA is created in the form of one residue of phosphoric 
acid. And two or three residues of phosphoric acids with aromatic bonds with carbon of sugar are already energy transporters. Nucleotides are the most important part of organic compounds of non-protein nature. They are the components of enzymes and other biologically active substances; they also serve as vectors of energy in the cells. Every nucleotide in DNA has the same phosphate group, the same sugar (deoxyribose) but there are four alternative bases: Adenine (A), Guanine (G), Cytosine (C) and Thymine (T). A single DNA molecule is actually made up of two chains of nucleotides joined together. These two polynucleotide chains line up alongside one another and are held together by hydrogen bonds between the pairs of bases in the middle of the molecule. There are two antiparallel sugar-phosophate backbones on the outside and the pairs of bases in the middle. You can see that the bases always pair together in a predictable way. A pairs with $\boldsymbol{T}$ (joined by two hydrogen bonds) and $\boldsymbol{C}$ pairs with $\boldsymbol{G}$ (joined by three hydrogen bonds) (https://pmgbiology.com/2014/10/21/dna-structure-and-function-igcse-a-understanding/).

A living organism is provided by energy (glucose), as well as heat. Enzymatic systems operate in conditions of low temperature heat in the range $4-42^{\circ} \mathrm{C}$. Temperature if ice melting $\left(4^{\circ} \mathrm{C}\right)$ is lower temperature limit of the existence of water in the liquid phase. Namely melted water called "alive" is the lower limit activities of living beings. The upper thermal boundary this is a limit of adaptive capacity of cells. A cell of the differentiated organism when adapting to high temperature, synthesizes proteins of warm shock (chaperone). When it reaches $42^{\circ} \mathrm{C}$, their synthesis is terminated and the cell dies. Its optimum temperature has for each enzyme system. The third source of energy for living things this is the food, which includes three main components: proteins, fats, and carbohydrates. The energy of decay of these components is used. The decay (oxidation) is carried out in two phases. In the first stage all three component are decayed with the formation of the final product: acetyl-CoA, which is burned then in the mitochondria with the participation of $\mathrm{O}_{2}$. Energy is liberated with help of protons and electrons and is stored in the form of ATP.

Carbohydrates are of energy sources in living systems. Carbohydrates contain carbon, hydrogen and oxygen in the ratio 1:2: 1 . One of the most important carbohydrates can be considered the sugar (glucose) containing six carbon atoms $\left(\mathrm{C}_{6} \mathrm{H}_{12} \mathrm{O}_{6}\right)$. Glucose is the product of photosynthesis and the basis of the food chain in the biosphere. The energy is stored when solar UV-photon has excited electron of carbon or phosphorus atoms. Complex carbohydrates are obtained by combining glucose, which are basic construction modules.

\section{THE CREATION OF BIOMOLECULES IN LIVE' ORGANISMS}

The creation of all the major biomolecules is made from a set of simple molecules (modules). This was supposed to be and the evolutionary path to large molecules. Indeed, at the beginning of the evolutionary way does not need to build very complex molecules. Over time could be added new modules, expanding functions and the set of large heterogeneous elements. The design of large biological molecules is made from the set of modules. The basis structural entities of proteins are amino acids. Like beads strung on a thread, these amino acids are collected to different sequences (different proteins). The hydrogen ion $\left(H^{+}\right)$one amino acid is combined with the hydroxyl $\left(\mathrm{OH}^{-}\right)$of other amino acid with the formation of water molecule.
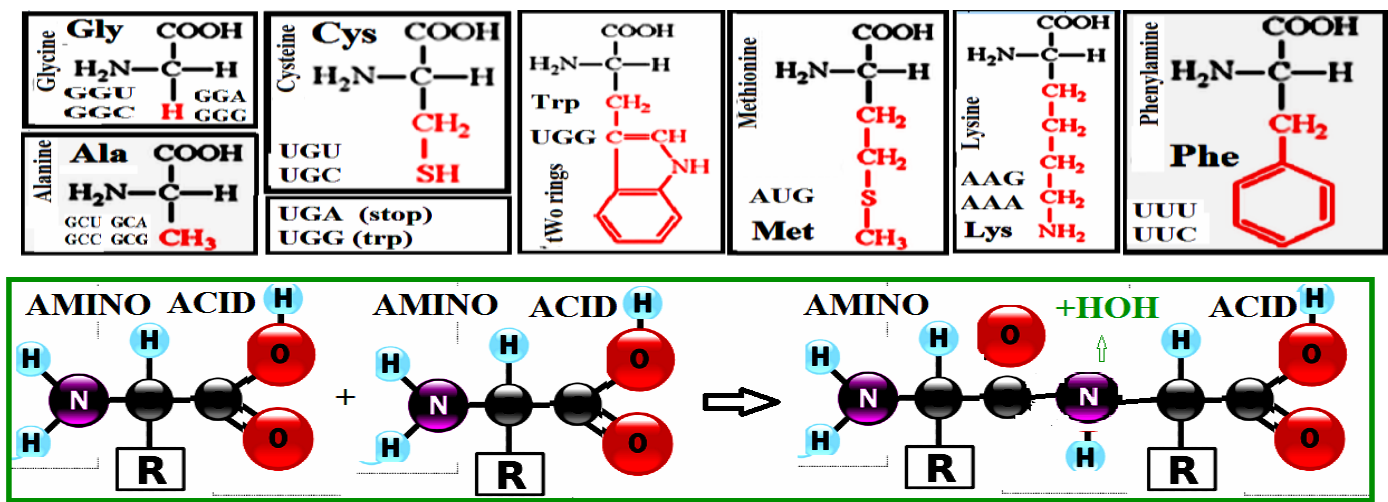

Fig.3. The common seeing of the amino acid molecules (down panel, left). Amino and acid groups are service and standard groups of all amino acids. They serve for the connection of different amino acids by each other (down panel, right). The radical R (red color in upper panel) provides functional properties and operational capabilities of the amino acid (https://ru.wikipedia.org/wiki/Amino_acids).

The DNA or RNA, which coding usually to enable only a single amino acid. Sequence of codons in the gene determines the sequence of amino acids in the polypeptide chain of the protein that encoded by the gene. There are four different nucleotides (adenine, guanine, cytosine and thymine). The total numbers of codons equals 64 , 61 of which encode different amino acids and 3 codons (UGA, UAG and UAA) signal to stop the broadcast of 
the polypeptide chain are called stop-codons. Stop codons are the sign of a complete build polypeptide chains. There are stop codons, which cause compulsory cessation of synthesis, others are conditional. The start codon in eukaryotic organisms is the triplet AUG in mRNA that encodes methionine, from which begins the translation process of a polypeptide chain. The start codons of some prokaryotes are also GUG, AUU, UUG and CUG codons. In the protein biosynthesis in the polypeptide chain is involved only 20 amino acids. So, codons can encode the same amino acid codons. Such codons called isoaccept codons.

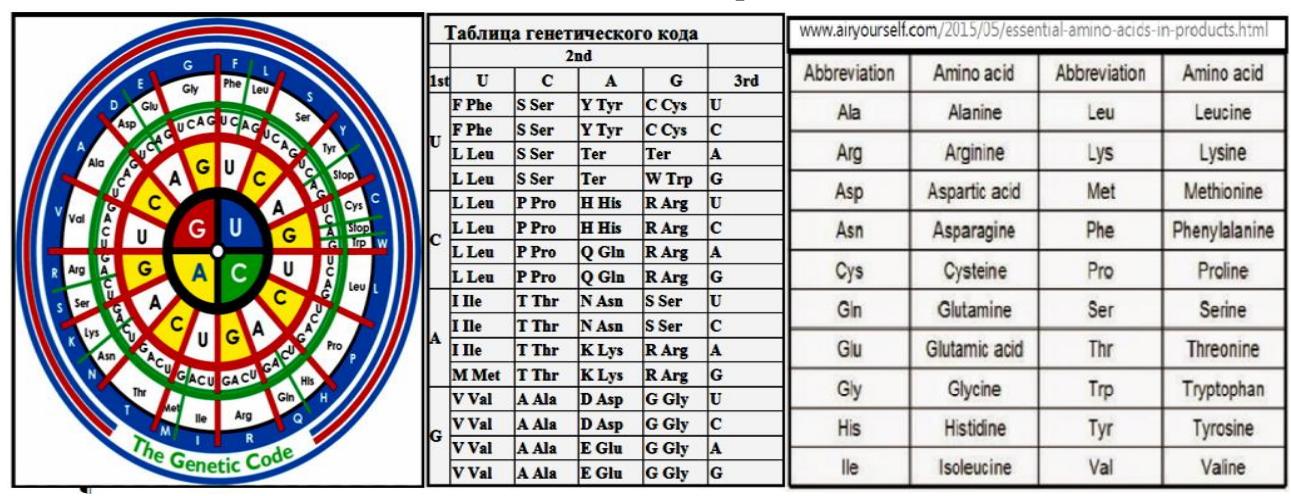

Fig.4. The coding manner of genetic information in the DNA created on the base of nucleotides.

The table of nucleotides treads consisted the full set of amino acids for creation of all proteins. http://www.airyourself.com/2015/05/essential-amino-acids-in-products.html

The codon (Threenucleotid) is the unit of the genetic code, three nucleotide residues (triplet) in the

The proteins, carbohydrates, and lipids are the food of living entities. Namely the food we must to produce from waste in settlements outside the Earth. The basis structural unit of the proteins is molecules of amino acids. These are a chain of atoms, which on the one hand goes outward the hydrogen atom of the water molecule, on the other hand its hydroxyl group. In the middle are a variety of other components. Of these amino acids are going to squirrel-hydrogen ion $(\mathrm{H}+)$ one amino acid is combined with the hydroxyl ion $(\mathrm{OH}-)$ other amino acids with the formation of water molecules. Among proteins there are proteins-enzymes that regulate chemical reactions in cells. And all proteins are the most important structures of living organisms.

Proteins are important structural components of living organisms. All life on Earth, from viruses to men, consists of proteins. In their diversity of proteins exceed all other macromolecules that are part of living organisms. Namely proteins are by these molecular machines $(M M)$ that perform all necessary operations in any living entity. There are proteins-enzymes, which catalyses chemical reactions in the cells. There are protective proteins, regulatory, structural and transport proteins. So, lots of immunoglobulin or antibodies that protect the body from invading bacteria and viruses in it. Other globulins are helping fix the damage to the blood vessels.

The amino acids join together to form a protein molecule. In the genetic code there are start and stop codons (nucleotide triplets). The AUG codon is universal start codon (also codes the methionine Met). Sometimes these are GUG or UUG codons. The stop codons UGA, UAA and UAG signal a termination of translation. While start codons need nearby sequences or initiation factors to start translation, a stop codon alone is sufficient to initiate termination Proteins are based on polypeptides, which are unique sequences of amino acids. Most codons in messenger RNA (from DNA) correspond to the addition of an amino acid to a growing polypeptide chain, which may ultimately become a protein. Stop codons signal the termination of this process by binding release factors, which cause the ribosomal subunits to disassociate, releasing the amino acid chain.

\section{PRODUCTION OF AMINO ACIDS}

Biosynthesis is the process of synthesis of natural organic compounds by living organisms, as the sequence of enzymatic (genetically determined) reactions. Occasionally, there are and spontaneous reactions, which go on without enzymatic catalysis. For example, during the leucine biosynthesis, one of the reactions is spontaneous and occurs without the participation of the enzyme. And biosynthesis of the same connections can go different ways from the same or from different source connections (https://ru.wiktionary.org/wiki/biosynthesis). Processes of biosynthesis play the exceptional role in all living cells. Protein biosynthesis is the multi-stage synthesis process of polypeptide chains of amino acids residues that occurs on the Ribosome in cells of living organisms involving tRNA and mRNA molecules.

The carbohydrates play a supporting role in cells, as included in the cell structure. So, vegetable fibers (cellulose) composed of chains of glucose molecules, which linked each other. The term is most common in biochemistry, where it is a synonym of saccharine, a group that includes sugars, starch, and cellulose. The saccharine are divided into four chemical, and polysaccharides. In general, the monosaccharide and 
disaccharides, which are smaller (lower molecular weight) carbohydrates, are commonly referred to as sugars. The names of the monosaccharide and disaccharides often end in the suffix -ose. For example, grape sugar is the monosaccharide glucose, cane sugar is the disaccharide sucrose, and milk sugar is the disaccharide lactose.

Carbohydrates contain oxygen, hydrogen and carbon in the ratio 1:2:1. In many living systems, carbohydrates are the energy sources. Critical carbohydrate can be considered a sugar-glucose $\left(\mathrm{C}_{6} \mathrm{H}_{12} \mathrm{O}_{6}\right)$. Glucose is the end product of photosynthesis and the base of the food chain in the biosphere. Joining molecules of glucose, as the main construction modules, you can get complex carbohydrates. Like proteins, carbohydrates play also the important role in cells, so as they included in the cell structure. Any fibers consist of chains of glucose molecules linked each other.

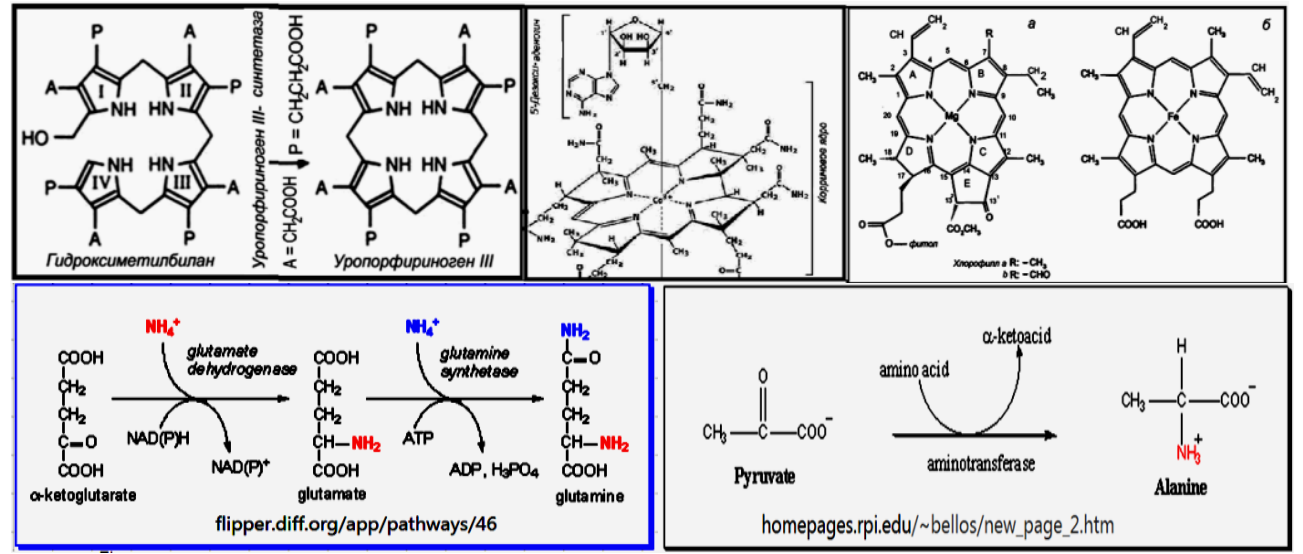

Fig.5. Molecular machines (MM) in the base of $\mathrm{Co}^{3+}$ ion create in cells and other $\mathrm{MM}$, for example, the hemoglobin molecules. In this case, energy of solar photons use also.

(https://books.google.com.ua/books?isbn=5457627039). Down panel: Ways of biosynthesis of some amino acids with MM aminotraspheraza. http://biochemistry.ru/biohimija_severina/B5873Part73-491.html

Lipids are organic molecules, which insoluble in water. Generally, hydrogen of water is universal link method in the live Nature. Lipids in living organisms perform two important functions. One class of molecules, phospholipids, consist of a small head that contains a phosphate group (the phosphorus atom, connected with four oxygen atoms), and the long hydrocarbon tail. The hydrocarbon tail of this molecule is hydrophobic. Energy state of the molecule is minimal when the tail is not in the water. Phosphate same of the head will be of hydrophilic, the energy state of the molecule is minimal in contact of the head with water. Phospholipids molecules seek to attain the minimum energy state, if we put them in water. They will line up in such a way that their tails will be together and heads are apart. This two-layer structure is very stable because the heads will be in contact with water, but the water is expelled from the area surrounding tails molecules. To move, lipid molecules require energy as for the removing of sites from water and for placing the hydrophilic or hydrophobic sites in water. Such double-layer structures of lipids have created cell membranes and walls inside the cell. These layers separated living matter from non-living matter. Lipids accumulate energy twice as much per unit of weight than carbohydrates. Live organisms store energy for the future in the form of lipids (fats).

In atomic physics, photons are radiated (or absorbed) of well-defined portions of energy in the transition between quantum levels. Every interaction between quantum systems is characterized by their specific set of conditions, their both discrete and continuous spectra. Directed movements, rotational and vibration degrees of freedom are reflected in spectra. The spectra, which have arisen for systems of atoms are most simple. So, the hydrogen atom has the discrete radiation of separate energetic states and also continuous spectra radiation of negative hydrogen ion $\left(6-1510^{3} \mathrm{~K}\right)$. In these spectra are only reflected the collisions between atoms, electrons and photons. This takes into account only the energy structure of quantum levels.

The live Nature can divided absorbed and stocked UV photon on some infrared photons (heat photons already). Here we can see the full knowledge of corpuscular-wave dualism of particles. This happens in the Krebs cycle, for example, using a set of various acids. The live Nature shows also its skill in convert of stored photons into heat, electricity and light. The spectra of molecules $\left(0-0.510^{2}{ }^{\circ} \mathrm{C}\right)$ have complex spectra of both lines and continuous bands. The role of the directed movements decreases with increasing size of the molecule and the growing role of rotational and vibration degrees of freedom. Here only these degrees of freedom are effective. But, most importantly, becomes the defining not only the electromagnetic interaction between the nucleus of atoms and linked electrons, but also the interaction of these electrons between them. And then introduced and analyzed the system of atom orbitals. Namely system of orbitals provides insight into processes of the atoms linking in the molecule and operation with detailed management of these relationships in 
molecules. Only the orbitals system of the atoms provides insight into the systems and catalytic methods (and with molecular machines in wildlife technologies) of splitting and synthesis of molecules. Wildlife $\left(300^{\circ} \mathrm{K}\right)$ uses the intensity of the vibration motions of protein molecules to regulate the rate of reactions and to constructing of their structures.

\section{THE PORTION TRANSFER OF ENERGY WITH SPECIALIZING MOLECULES}

Transfer function of energy in a cell carries out an army of specialized molecules. The most important ones are adenosine triphosphate (ATP) and adenosine diphosphate (ADP). Both molecules are constructed like this: a known set of atoms of carbon, hydrogen and nitrogen (this adenine) attached to the ribose molecule (this sugar), and all this together is attached to the tail of the phosphate. Of the names of the molecules it is clear that in the tail of the ADP contains two phosphate and in the tail of ATP contains the three phosphate. In the process of photosynthesis, for example, energy goes to join the third phosphate to the tail of ADP. The resulting molecule of ATP then migrated to other parts of the cell and is used in other processes. It excels at peeling last phosphate in ATP turns into ADP again. Free energy is reduced by the DF value (quantum of energy). Energy dissipated as heat when the ATP synthesis absence. The ATP molecule is a versatile form of energy conservation of cells and its use at any time. The main supplier of energy into cells is glucose (carbohydrates). Energy is extracted in multi-stage manner of chemical ways. First, in the cytoplasm of the cell and without oxygen, the molecule of glucose is destroyed into two molecules of pyruvic acid (glycolysis). The $50 \mathrm{kcal} / \mathrm{mol}$ of energy is released ( 7\% of glucose energy). Part of it is dissipated as heat; the other is spent on the creation of two molecules of ATP.In bioenergetics, where processes in cells occur in the absence of changes in temperature, pressure and volume, we call "soft" technologies. In physics, where the transition of heat to work is impossible, the heat is appear an irreversible loss of energy. In bioenergetics is applied a number of bio-specific mechanisms for direct conversion of one form of free energy to another without transition into heat. It is converted into a form of free chemical energy with special compounds, in which the energy is extremely mobile. It can to morph into other forms of energy and at a constant temperature, in particular to make work or used for biosynthesis with high efficiency ( $30 \%$ in muscle work). The uniformity had been established in energy processes throughout the living world, from micro-organisms to humans. For all plant and animal life were common and those matter in which the energy accumulated in mobile and biologically acceptable form and processes, by which such storage takes place. The same uniformity is installed and in the processes of use of stored energy for those substances. The uniformity take place in the structure of proteins and contractile mechanism of transformation of chemical energy into work the same when moving flagella at the simplest, lowering the mimosa leaves and in the movements of birds and mammals. Such uniformity is characteristic and to store and transmit hereditary information, ways of biosynthesis and enzymatic reactions.

\section{OTHER METHODS OF THE ORGANICS OPERATION}

Mankind has long mastered the technologies to use as a fuel of the plant organics of living Nature $\left(H_{n} C_{m} N_{j} O_{k}\right.$ molecules), the wood and fossil carbons (atomic structures of $C_{n}$ ) and hydrogen $\left(H_{2}\right)$. It's the burning (tying with oxygen). Even production of the charcoal is mastered, but with the losing of hydrogen (for example, Ostapenko, 2013, fig. 2.1.1). Particular environmental load gave rise to wide use of fossil fuels (the beginning of technical era since the 20 century). Pyrolysis, this is the thermal decomposition of organic and many other compounds at oxygen lack (especially wood and petroleum products). Always, this is the decomposition of any connections into smaller molecules by the action of high temperature (typically $\sim 700^{\circ} \mathrm{C}$ and more). But the Telluride decomposes into hydrogen and tellurium ${ }^{128} \mathrm{Te}_{52}\left([\mathrm{Kr}] 4 \mathrm{~d}^{10} 5 \mathrm{~s}^{2} 5 \mathrm{p}^{4}\right)$ is already at a temperature of $\sim 0^{\circ} \mathrm{C}$. The electro-discharge technologies of organics destruction are connected with yet more high temperatures and they are energy costs. In addition, nitrogen oxides and dioxins arisen also.

Our technology to produce still and hydrogen from the organics do not make additional heat load to the atmosphere, but they increased doubled the amount of fuel. The simple burning of the hydrogen fuel is the first face of the hydrogen energetics (for example, fig. 2.1.1d). The new $E F h E F$ energetics allows us to embed in the practice of terrestrial farming the technologies of decomposition of the organics, with the subsequent synthesis of primary energy resources. Thus, we replaced the fossil energy sources, at the first stage, and then we may to move on to the production of other organic substances and materials. Using "solar" radiation of negative hydrogen ions, we may to master the technologies of livelihood support in autonomous and isolated settlements. When we constructed of residential and industrial buildings we can to give their ability to function in time of global catastrophes. For the first time it became possible to speak about this after the establishment of the theory (Ostapenko, 2011) of the managed energy release of negative hydrogen ions, which is identical to the continuous spectrum of radiation from the photosphere of the Sun. Namely these photons of solar radiation have used the fauna on Earth to create of organic matter through photosynthesis. And the living Nature has mastered "soft" technologies using in detail all quantum-mechanical properties of particles, atoms and their compounds. 
Life support systems are required to evolve on the Earth today already, if we want to preserve the ecological balance of the Earth, if we want to master remote, waterless, permafrost areas and underwater areas. The success can only come through long periods of time on the basis of the restructuring of the entire economic system. This is yet more important in the development of other planets with totally unsuitable and hostile medium for terrestrial organisms.

The first objective of the creators of the "Biosphere" project was to prove that modern earthlings can create space colony and be in an enclosed space. The crew of the "Biosphere" was conquered by colonies of cockroaches and ants. It shows that science doesn't always achieve results with the first time. But in the autonomous settlements, especially on other planets and interstellar expeditions, such failures are not allowed. And we still know nothing about the possible evolution of terrestrial life forms in their extended stay in conditions of autonomous space settlements.

As base energy source is logically to use nuclear power (electricity and heat) and physical power of hydrogen photons. All other energy sources will disappear during a global catastrophe. Even for thermonuclear sources the replace may be found (for example, Burkay Kemal, 2016). These are three dimensional penetrating functions from electro-magnetic radiation to super high heat energy and electric current with zero mass loss. But, only the physical energy of the photons of negative hydrogen ions (second face of hydrogen power) will provide people with energy and light, and will also return all $100 \%$ organic waste in a new cycle of use. The refinement of the technologies of survival it is advisable to produce on the Earth by creating a network of autonomous settlements of different specialization: at ground level, underground, underwater, and then in space stations and planets of the solar system. These technologies will be in demand in settlements outside the Earth. The source of light and heat can now be at the disposal of people in any environment on Earth and at any distance from the Sun in outer space.

\section{NEW SYSTEMS OF LIGHTING AND HEATING}

People are capable already to build autonomous settlements, which should be with the light and warm, while providing the comfortable environment for flora and fauna. In space is already going to mirrors, which will illuminate the entire regions of the Earth's surface in ten times brighter than the full moon. For lighting can be used direct energy from the Sun. This will increase the efficiency of solar stations on Earth and would prolong the growing period of plants. This lighting system is not afraid of earthly cataclysms.

Many components and technologies, to create "artificial Sun" of hydrogen energetics, already developed and debugged in the industry. So, the X-ray lamps are equipped by systems of electron flows for excitation of UV mercury lines $(184.9499,253.6517,435.8328 \mathrm{~nm}$ ) in plasma glow bulb (section 1-7.10). These emission lines are in the range of the light sensitivity of chlorophyll for plant growth. Namely such electron flows and line radiation are required for excitation of the continuous spectrum of negative hydrogen ion in plasma (fig. 1-3.7.2). To obtain the desired flow of continuous radiation, densities of hydrogen atoms are needed $>10^{18} \mathrm{~cm}^{-3}$ that on the Earth it's yet a deep vacuum. The problem of creating a "solar" photon of need range energies is achieved in reactors of our installations of decomposition/synthesis of the organics. And then we may to provide the lighting, water and air regeneration and "soft" technologies of work with organic, in the tasks of obtaining primary energy sources and also we may to ensure the full cycle of life activity of autonomous and remote settlements. For more global illumination of the planet or individual regions can apply experience of magnetic plasma confinement in the TOKOMAK and ITER installations (fusion power, section 5-7.3), and the experience of the use of hydrogen-powered tablets and their laser ignition of the man-made "California Sun" (Ostapenko, 2013, section 1-7.4).

\section{CONCLUSIONS}

It is developed the bases of new physical (atoms) energy, which is placed in one row with the chemical energy (molecules) and quantum (nuclear). Eco Physical hydrogen Energetics of Photons (EFhEF-energetics) of physical decomposition/synthesis of organic operates with the energy of individual photons. Analogues of technology are absent yet. But, this energy is mastered (photosynthesis plants) to create living organisms on the Earth.

Firstly, photons of "solar" radiation can be produced by man regardless of the Sun. This is the new quality transition of mankind with the time of harnessing of fire. In the base of technology we use the process $\left(H_{n} C_{m} N_{j} O_{k}\right.$ +photon $\left.\rightarrow \mathrm{C}+\mathrm{H}_{2}\right)$. It is the reverse process of organic creating $\left(\mathrm{CO}_{2}+\mathrm{H}_{2} \mathrm{O}+\right.$ photon $\rightarrow H_{n} C_{m} N_{j} O_{k}$ $+\mathrm{O}_{2}$ ), which is going on Earth with the participation of a molecule of chlorophyll. The direct and reverse processes used the energy of the hydrogen negative ion.

A relief problem of synthesis of the complete set of proteins and other food products is existed. This can be "to give" the organism itself. Any live organism consuming organic food decomposes it to amino acids (to triplets of nucleotides). Food will be inconvenient or impossible nucleotides and then the goal is to get the right proteins using it is genetic engineering. 
The first installations of organic matter destruction with simultaneous production of the gas fuel were created. The new energetics might to replace the limited reserves of fossil energy (coal, oil, gas) of unlimited resources of wildlife. The EFhEF- energetics, which does not using the natural resources and oxygen, is only the first step on the path to creating a full cycle of ecological life support on Earth and in space. The EFhEF energetics allows us to "take" to the road and the Sun itself.

\section{REFERENCES}

[1] Ostapenko V. A. Solar flares: current sheets on the Sun, ecological hydrogen energetics on the Earth. (ru), Kiev, «Ukraine», 2011, 450 p. (ru).

[2] Ostapenko V. A. Solar flares: current sheets on the Sun, ecological hydrogen power on the Earth. (en), Kiev, "Ukraine", 2012, 185 p. (en, The main text of the doctoral thesis).

[3] Ostapenko V. A. Environmental hydrogen energetics. (ru), Kiev, «Ukraine», 2013, 335 p.

[4] Ostapenko V. A. Energetics of molecules, atoms, and nuclei (1.Energetics: physical and biophysical). Kiev, «Ukraine», 2014, v.1, 370 p. (ru)

[5] Ostapenko V. A. Energetics of molecules, atoms, and nuclei (II.Energetics: chemical and quantum). (ru), Kiev, «Ukraine», 2015, v.2, 292 p. (ru)

[6] 2016, Site: http://osdar.hol.es/.

[7] Averina N. G., Yaronskaya E. B. Biosynthesis tetrapyrrols in plants. Minsk, «Belarus' science», 2012, 413 p. (Ru), NAS of Belarus, Institute of biophysics and cell engineering. https://books.google.com.ua/books?isbn=5457627039

[8] Biochemistry. Red. E. S. Severina. Textbook for high schools. 2003, 779 p. (Ru). http://biochemistry.ru/biohimija_severina/B5873Content.html.

[9] Burkay Kemal, CEO. Generating super high heat energy from the electromagnetic radiation in special alloys. (2016), Quantum Dynamic Sciences Inc. (www.qdsciences.com).

[10] Shay Michael \& Matthaeus William. Plasma physics' giant leap. Project of Delaware University, May 12, 2016. http://www.sciencecodex.com/plasma_physics_giant_leap-182559 